\title{
Metanephric adenoma: the utility of immunohistochemical and cytogenetic analyses in differential diagnosis, including solid variant papillary renal cell carcinoma and epithelial-predominant nephroblastoma
}

Stephanie N Kinney ${ }^{1}$, John N Eble ${ }^{1}$, Ondrej $\mathrm{Hes}^{2}$, Sean R Williamson ${ }^{3}$, David J Grignon ${ }^{1}$, Mingsheng Wang ${ }^{1}$, Shaobo Zhang ${ }^{1}$, Lee Ann Baldrige ${ }^{1}$, Guido Martignoni ${ }^{4}$, Matteo Brunelli ${ }^{4}$, Lisha Wang ${ }^{5}$, Eva Comperat ${ }^{6}$, Rong Fan ${ }^{1}$, Rodolfo Montironi ${ }^{7}$, Gregory T MacLennan ${ }^{8}$ and Liang Cheng 1

${ }^{1}$ Department of Pathology and Laboratory Medicine, Indiana University School of Medicine, Indianapolis, IN, USA; ${ }^{2}$ Department of Pathology, Charles University Hospital Plzeñ, Pilsen, Czech Republic; ${ }^{3}$ Department of Pathology and Laboratory Medicine, Henry Ford Health System, Detroit, MI, USA; ${ }^{4}$ Dipartimento di Patologia, Universitá di Verona, Verona, Italy; ${ }^{5}$ Department of Pathology, Fudan University Shanghai Cancer Center, Shanghai, China; ${ }^{6}$ Department of Pathology, Groupe Hospitalier Pitié-Salpêtrière, Paris, France; ${ }^{7}$ Department of Pathological Anatomy and Histopathology, School of Medicine, Polytechnic University of the Marche Region (Ancona), Ancona, Italy and ${ }^{8}$ Department of Pathology and Laboratory Medicine, Case Western Reserve University, Cleveland, OH, USA

\begin{abstract}
Metanephric adenoma is a benign renal neoplasm that overlaps in morphology with the solid variant of papillary renal cell carcinoma and epithelial-predominant nephroblastoma. To aid in resolving this differential diagnosis, we investigated the utility of immunohistochemical and molecular analyses in distinguishing between these entities; the first study, to our knowledge, to use a combined approach in analyzing all three tumors. We analyzed 37 tumors originally diagnosed as metanephric adenomas $(2$ of which we reclassified as papillary renal cell carcinomas), 13 solid variant papillary renal cell carcinomas, and 20 epithelial-predominant nephroblastomas using a combination of immunohistochemistry and fluorescence in situ hybridization (FISH) assessing for trisomy of chromosomes 7 and 17 and loss of Y. Immunohistochemical staining was performed for CK7, AMACR, WT1, and CD57. The combination of CK7-, AMACR-, WT1+, and CD57+ was considered characteristic of metanephric adenoma. Most of the tumors originally diagnosed as metanephric adenomas (31/37) showed the expected staining pattern of metanephric adenoma (CK7 -, AMACR-, WT1+, and CD57+). Of the six tumors with discordant immunophenotype, two tumors were reclassified as papillary renal cell carcinoma after cytogenetic workup. It is recommended that all adult cases histologically resembling metanephric adenoma have WT1, CD57, CK7, and AMACR immunohistochemical staining performed. If the staining pattern is characteristic for metanephric adenoma (CK7-, AMACR-, WT1+, and CD57+, including membranous staining), then no other diagnostic tests are indicated. However, if there is a different immunostaining pattern, then we recommend FISH analysis.
\end{abstract}

Modern Pathology (2015) 28, 1236-1248; doi:10.1038/modpathol.2015.81; published online 7 August 2015

Correspondence: Dr L Cheng, MD, Department of Pathology and Laboratory Medicine, Indiana University School of Medicine, 350 West 11th Street, IUHPL Room 4010, Indianapolis, IN 46202, USA. E-mail: liang_cheng@yahoo.com

Received 18 February 2014; revised 4 March 2015; accepted 29 May 2015; published online 7 August 2015
Metanephric adenoma is an uncommon benign renal tumor that generally occurs in adults and occasionally children. ${ }^{1-3}$ Metanephric adenoma is the most common pediatric renal epithelial tumor. ${ }^{4}$ The patients are mainly women, with the mean age 
Table 1 Immunohistochemical reagents and sources

\begin{tabular}{llll}
\hline Reagent & Source & Pretreatment & Dilution \\
\hline AMACR (P504S) & Dako Corporation, Carpinteria, CA, USA & TRS high pH & Ready to use \\
WT1 & Dako Corporation, Carpinteria, CA, USA & TRS high pH & Ready to use \\
CK7 & Dako Corporation, Carpinteria, CA, USA & TRS low pH & Ready to use \\
CD57 & Dako Corporation, Carpinteria, CA, USA & TRS low pH & Ready to use \\
\hline
\end{tabular}
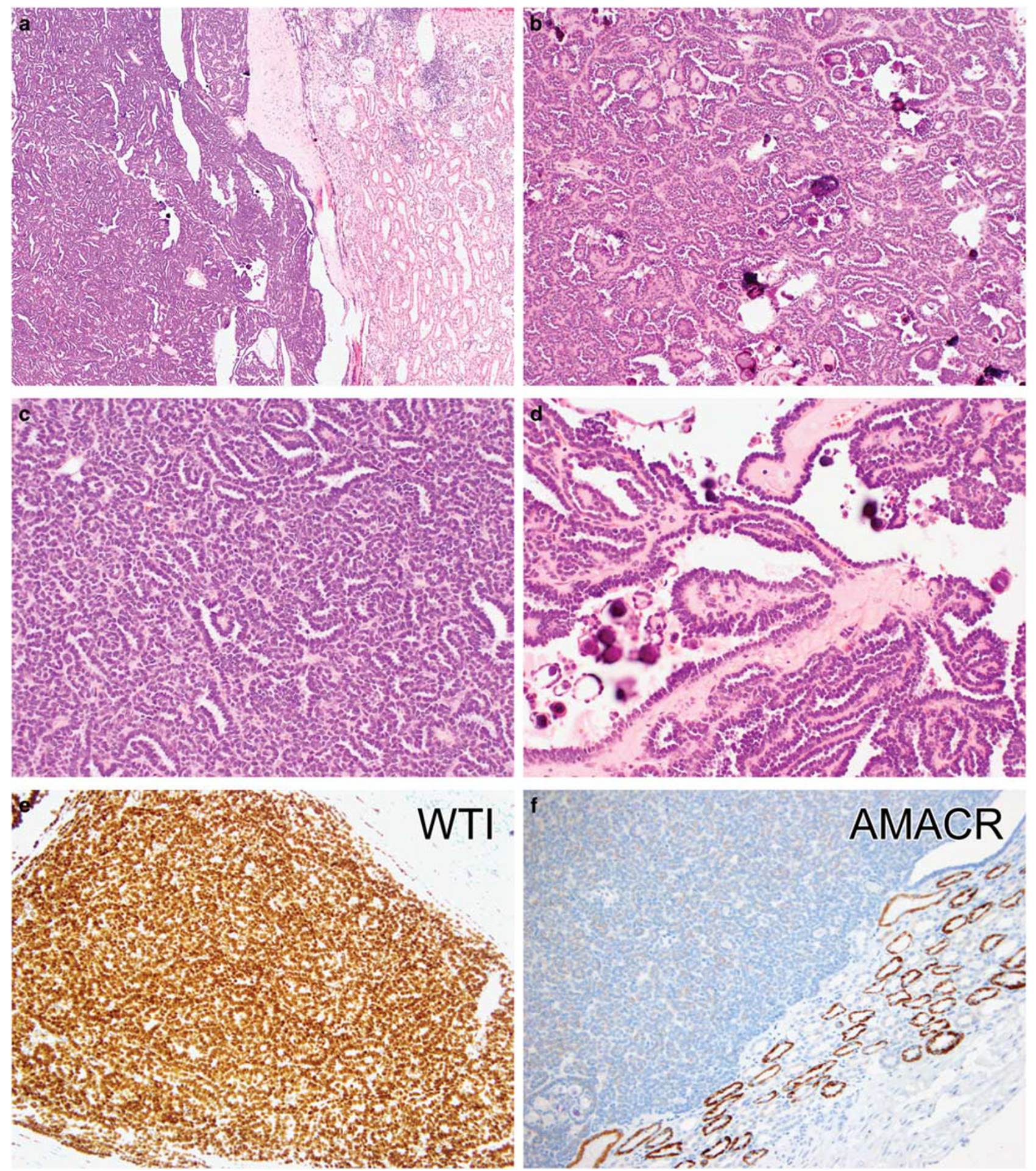

Figure 1 For caption see page 1238. 
being 41 years. ${ }^{1}$ The differential diagnosis of metanephric adenoma can be categorized based on age. In children (12 years and younger), metanephric adenoma can have similar morphology to epithelialpredominant nephroblastoma. In adults ( $\geq 30$ years), metanephric adenoma overlaps in morphology with papillary renal cell carcinoma. Prior immunohistochemical studies of metanephric adenoma have shown characteristic staining patterns of negativity for CK7 and AMACR and positivity for WT1 and CD57. ${ }^{1-3,5,6}$ Other studies have shown papillary renal cell carcinoma staining patterns of negativity for WT1 and CD57 and positivity for AMACR and CK7.5,6 Nephroblastoma has been shown to characteristically stain positively for WT1. ${ }^{7,8}$ Likewise, cytogenetic studies have shown that metanephric adenoma lacks the gains of chromosome 7 and 17 and loss of $Y$ that are characteristic of papillary renal cell carcinoma. ${ }^{9-12}$ To aid in resolving this differential diagnosis, we investigated the utility of combined immunohistochemical and molecular analyses of 37 tumors originally diagnosed as metanephric adenomas (2 of which we reclassified as papillary renal cell carcinomas), 13 solid variant papillary renal cell carcinomas (plus the 2 reclassified tumors), and 20
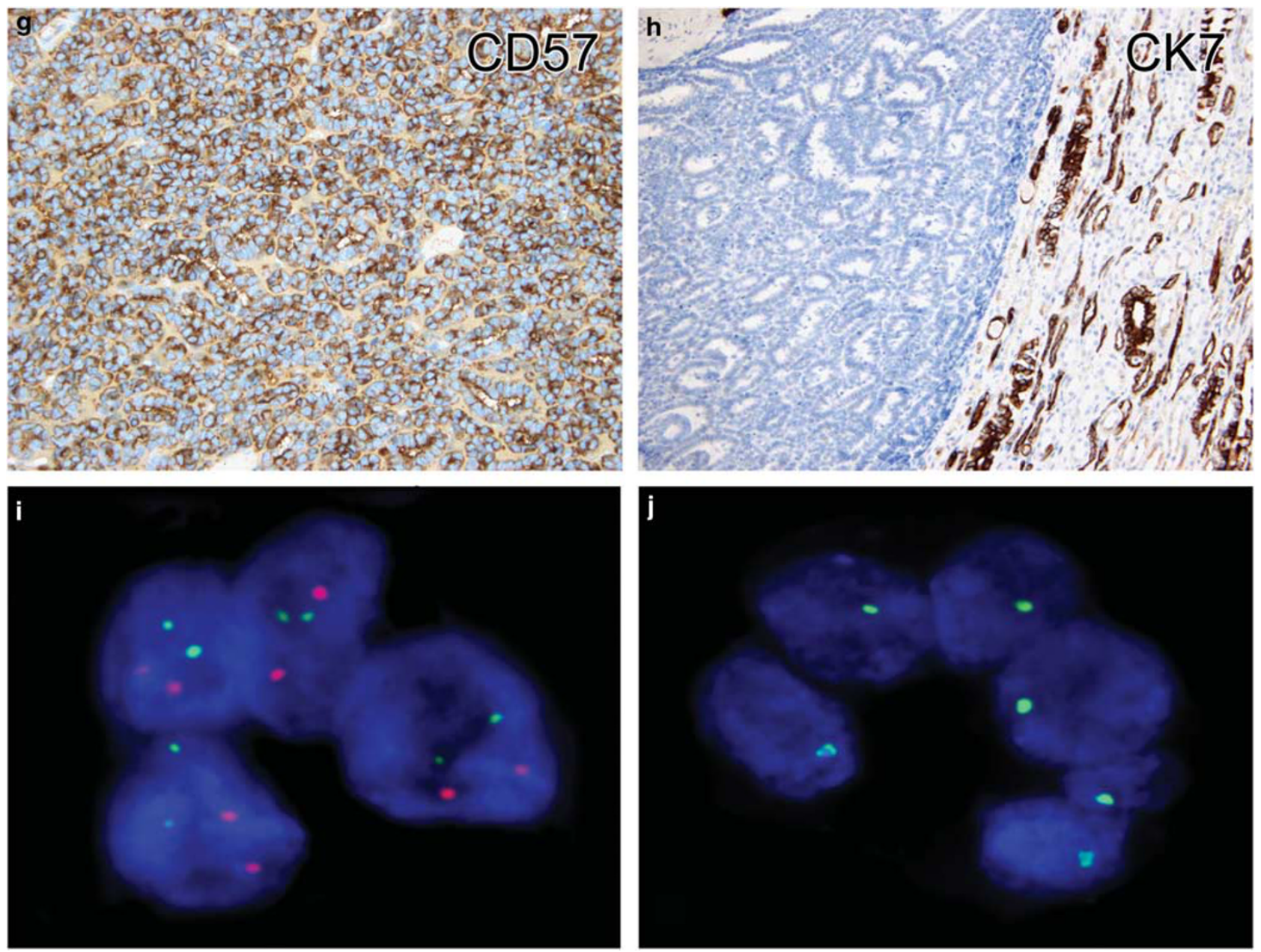

Figure 1 Continued.

Figure 1 Histology and immunohistochemistry of metanephric adenoma. (a) Distinct interface between tumor and uninvolved renal parenchyma (H\&E). (b) Papillary structures with dystrophic calcification. (c) Tightly packed acini. (d) Papillary structures with more edematous stroma. (e) WT1 shows nuclear positivity. (f) AMACR is negative in tumor cells and positive in normal renal tubules. (g) CD57 shows cytoplasmic positivity. (h) CK7 is negative in tumor cells and positive in normal renal tubules. (i) Fluorescence in situ hybridization with centromeric probes for chromosomes 7 (red signal) and 17 (green signal) show normal nuclei with two red and two green signals. (j) Fluorescence in situ hybridization with centromeric probe for the Y chromosome (green signal) shows normal nuclei with one green signal. 
Table 2 Clinical, pathological, immunohistochemical, and molecular findings

\begin{tabular}{|c|c|c|c|c|c|c|c|c|c|c|c|}
\hline Case \# & $\begin{array}{l}\text { Age, } \\
\text { years }\end{array}$ & Gender & $\begin{array}{c}\text { Tumor } \\
\text { size }(\mathrm{cm})\end{array}$ & WT1 & $A M A C R$ & CK7 & CD57 & Ch 7 & Ch 17 & Ch $Y$ & Diagnosis \\
\hline 1 & 49 & M & 1.6 & Diffuse & Neg & Neg & Diffuse & Disomy & Disomy & Normal & MA \\
\hline 2 & 70 & $\mathrm{~F}$ & 4.2 & Diffuse & Neg & Neg & Diffuse & Disomy & Disomy & NA & MA \\
\hline 3 & 46 & $\mathrm{M}$ & 5 & Diffuse & Neg & Neg & Diffuse & Disomy & Disomy & Normal & MA \\
\hline 4 & 60 & $\mathrm{~F}$ & 2.4 & Diffuse & $\mathrm{Neg}$ & Neg & Diffuse & Disomy & Disomy & NA & MA \\
\hline 5 & 12 & $\mathrm{M}$ & 6.5 & Diffuse & Neg & Neg & Diffuse & Disomy & Disomy & Normal & MA \\
\hline 6 & 7 & $\mathrm{M}$ & 3.4 & Intermediate & Neg & Neg & Diffuse & Disomy & Disomy & Normal & MA \\
\hline 7 & 71 & $\mathrm{M}$ & 1.5 & Diffuse & $\mathrm{Neg}$ & Neg & Diffuse & Disomy & Disomy & Normal & MA \\
\hline 8 & 49 & $\mathrm{~F}$ & 4 & Diffuse & Neg & Neg & Diffuse & Disomy & Disomy & NA & MA \\
\hline 9 & 74 & $\mathrm{~F}$ & 3.5 & Diffuse & Neg & Neg & Diffuse & Disomy & Disomy & NA & MA \\
\hline 10 & 53 & $\mathrm{~F}$ & 4.6 & Diffuse & $\mathrm{Neg}$ & Neg & Diffuse & Disomy & Disomy & NA & MA \\
\hline 11 & 66 & $\mathrm{~F}$ & 5 & Diffuse & Neg & Neg & Diffuse & Disomy & Disomy & NA & MA \\
\hline 12 & 63 & $\mathrm{~F}$ & & Diffuse & Neg & Neg & Diffuse & Disomy & Disomy & NA & MA \\
\hline 13 & 61 & M & 4 & Diffuse & $\mathrm{Neg}$ & Neg & Diffuse & Disomy & Disomy & Normal & MA \\
\hline 14 & 84 & $\mathrm{~F}$ & 2.5 & Diffuse & Neg & Neg & Diffuse & Disomy & Disomy & NA & MA \\
\hline 15 & 23 & M & 1.1 & Diffuse & Neg & Neg & Diffuse & Disomy & Disomy & Normal & MA \\
\hline 16 & 74 & $\mathrm{~F}$ & 4 & Diffuse & $\mathrm{Neg}$ & Neg & Diffuse & Disomy & Disomy & NA & MA \\
\hline 17 & 47 & $\mathrm{~F}$ & 4.3 & Diffuse & Neg & Neg & Diffuse & Disomy & Disomy & NA & MA \\
\hline 18 & 33 & $\mathrm{~F}$ & 2 & Diffuse & Neg & Neg & Diffuse & Disomy & Disomy & NA & MA \\
\hline 19 & 52 & $\mathrm{~F}$ & 2.2 & Diffuse & $\mathrm{Neg}$ & Neg & Diffuse & Disomy & Disomy & NA & MA \\
\hline 20 & 81 & $\mathrm{~F}$ & 1.8 & Diffuse & Neg & Neg & Diffuse & Disomy & Disomy & N/A & MA \\
\hline 21 & 76 & $\mathrm{~F}$ & 7 & Diffuse & Neg & Neg & Diffuse & Disomy & Disomy & NA & MA \\
\hline 22 & 70 & M & 3 & Diffuse & Neg & Neg & Diffuse & Disomy & Disomy & Normal & MA \\
\hline 23 & 48 & $\mathrm{~F}$ & 1.5 & Diffuse & Neg & Neg & Diffuse & Disomy & Disomy & NA & MA \\
\hline 24 & 20 & $\mathrm{~F}$ & 3 & Diffuse & Neg & Neg & Diffuse & Disomy & Disomy & NA & MA \\
\hline 25 & 63 & M & 7 & Diffuse & $\mathrm{Neg}$ & Neg & Diffuse & Disomy & Disomy & Normal & MA \\
\hline 26 & & $\mathrm{~F}$ & & Diffuse & Neg & Neg & Diffuse & Disomy & Disomy & NA & MA \\
\hline 27 & 33 & $\mathrm{~F}$ & 1.5 & Diffuse & Neg & Neg & Diffuse & Disomy & Disomy & NA & MA \\
\hline 28 & 43 & $\mathrm{~F}$ & 4.5 & Intermediate & Neg & Neg & Diffuse & Disomy & Disomy & NA & MA \\
\hline 29 & 54 & $\mathrm{~F}$ & 3.9 & Diffuse & Neg & Neg & Diffuse & Disomy & Disomy & NA & MA \\
\hline 30 & 48 & $\mathrm{~F}$ & 2 & Intermediate & Neg & Neg & Diffuse & Disomy & Disomy & NA & MA \\
\hline 31 & 30 & M & 4 & Diffuse & Neg & Neg & Diffuse & Disomy & Disomy & Normal & MA \\
\hline 32 & 10 & M & 2.6 & Diffuse & Neg & Neg & $\begin{array}{l}\text { Diffuse } \\
\text { (membranous) }\end{array}$ & Disomy & Disomy & Normal & MA \\
\hline 33 & 56 & $\mathrm{~F}$ & 1.4 & Diffuse & Neg & Neg & $\begin{array}{l}\text { Diffuse } \\
\text { (membranous) }\end{array}$ & Disomy & Disomy & NA & MA \\
\hline 34 & 48 & $\mathrm{~F}$ & 3 & Diffuse & Neg & Neg & $\begin{array}{l}\text { Diffuse } \\
\text { (membranous) }\end{array}$ & Disomy & Disomy & NA & MA \\
\hline 35 & 60 & $\mathrm{~F}$ & 5 & Diffuse & Focal & Neg & Diffuse & Disomy & Disomy & NA & MA \\
\hline $\mathrm{A}^{\mathrm{a}}$ & 69 & M & 1.9 & Neg & Diffuse & Diffuse & Focal & Disomy & Disomy & Deletion & PRCC \\
\hline $\mathrm{B}^{\mathrm{a}}$ & 83 & M & 2 & Neg & Diffuse & Diffuse & Neg & Trisomy & Trisomy & Deletion & PRCC \\
\hline
\end{tabular}

Abbreviations: Diffuse, $>50 \%$; Focal, 1-25\%; Intermediate, 26-50\%; MA, metanephric adenoma; NA, not applicable; Neg, negative ( $<1 \%)$; PRCC, papillary renal cell carcinoma.

${ }^{\mathrm{a}}$ The two tumors we reclassified as PRCC.

epithelial-predominant nephroblastomas in distinguishing between these entities; the first study, to our knowledge, to use a combined approach for all three tumor types.

\section{Materials and methods}

Thirty-seven tumors originally diagnosed as metanephric adenomas (2 of which we reclassified as papillary renal cell carcinomas), 13 solid variant papillary renal cell carcinomas (plus the 2 reclassified tumors), and 20 epithelial-predominant nephroblastomas were identified from the surgical pathology files of the participating institutions. Tumors from a previous study were not included. ${ }^{9}$ All original slides were reviewed. Unstained $4-\mu \mathrm{m}$ sections were cut from the paraffin block selected for each case and deparaffinized by routine techniques. Antibodies directed against CK7 (monoclonal mouse antihuman CK7 antibody, ready to use; DAKO), CD57 (monoclonal mouse antihuman CK7 antibody, ready to use; DAKO), WT1 (monoclonal mouse antihuman CK7 antibody, ready to use; DAKO), and AMACR/P504S (monoclonal rabbit antihuman antibody, ready to use, DAKO) were utilized in a DAKO automated instrument (Table 1). All stains were performed using the FLEX EnVision plus Autostainer system. Positive and negative controls gave appropriate results for each procedure.

The extent of immunohistochemical staining was evaluated microscopically. A tumor was considered positive if it showed staining from 1 to $100 \%$ and negative if there is no staining $(0 \%)$. Positivity 
Table 3 Summary of immunohistochemical staining properties in metanephric adenoma, papillary renal cell carcinoma, and epithelialpredominant nephroblastoma

\begin{tabular}{|c|c|c|c|c|}
\hline Antibody & Staining characteristics ${ }^{\mathrm{a}}$ & $\begin{array}{c}\text { Metanephric adenoma, } \\
\mathrm{n}=35^{\mathrm{b}}(\%)\end{array}$ & $\begin{array}{c}\text { Papillary renal cell } \\
\text { carcinoma, } \mathrm{n}=15^{\mathrm{c}}(\%)\end{array}$ & $\begin{array}{c}\text { Epithelial-predominant } \\
\text { nephroblastoma, } \mathrm{n}=20(\%)\end{array}$ \\
\hline \multirow[t]{4}{*}{ WT1 } & Negative & 0 & $15(100)$ & 0 \\
\hline & Focal & 0 & 0 & 0 \\
\hline & Intermediate & $3(9)$ & 0 & $2(10)$ \\
\hline & Diffuse & 32 (91) & 0 & $18(90)$ \\
\hline \multirow[t]{4}{*}{ AMACR } & Negative & $34(97)$ & 0 & $20(100)$ \\
\hline & Focal & $1(3)$ & 0 & 0 \\
\hline & Intermediate & 0 & 0 & 0 \\
\hline & Diffuse & 0 & $15(100)$ & 0 \\
\hline \multirow[t]{4}{*}{ CK7 } & Negative & $35(100)$ & $1(7)$ & $19(95)$ \\
\hline & Focal & 0 & 0 & 0 \\
\hline & Intermediate & 0 & 0 & $1(5)$ \\
\hline & Diffuse & 0 & $14(93)$ & 0 \\
\hline \multirow{4}{*}{ CD57 } & Negative & 0 & $14(93)$ & $4(20)$ \\
\hline & Focal & 0 & $1(7)$ & $10(50)$ \\
\hline & Intermediate & 0 & 0 & $2(10)$ \\
\hline & Diffuse & $35(100)$ & 0 & $4(20)$ \\
\hline
\end{tabular}

${ }^{\mathrm{a}}$ Scoring: Diffuse $>50 \%$, intermediate $26-50 \%$, focal $1-25 \%$, negative $<1 \%$. ${ }^{\mathrm{b}}$ Two tumors were reclassified as papillary renal cell carcinoma and were excluded from analysis with metanephric adenomas. ${ }^{\mathrm{C}}$ The two reclassified tumors were analyzed in the papillary renal cell carcinoma category.

was scored as diffuse if $>50 \%$ of the tumor cells stained positively; positive staining between 26 and $50 \%$ was scored as intermediate, between 1 and $25 \%$ scored as focal, and below this threshold tumors were scored as negative.

Fluorescence in situ hybridization (FISH) analyses were performed as described previously. ${ }^{13-18}$ Briefly, multiple 4- $\mu$ m sections were obtained from formalin-fixed paraffin-embedded tissue blocks containing neoplastic tissue. A hematoxylin and eosinstained slide from each block was examined to identify areas of neoplastic tissue for FISH analysis. The slides were deparaffinized with two washes of xylene, $15 \mathrm{~min}$ each, and subsequently washed twice with absolute ethanol, $10 \mathrm{~min}$ each, and then air dried in a fume hood. Next, the slides were treated with $0.1 \mathrm{mM}$ citric acid ( $\mathrm{pH}$ 6.0) (Zymed, South San Francisco, CA, USA) at $95^{\circ} \mathrm{C}$ for $10 \mathrm{~min}$ and rinsed in distilled water for $3 \mathrm{~min}$, followed by a wash with $2 \times$ standard saline citrate for 5 min. Digestion of nuclear proteins was performed by applying $0.4 \mathrm{ml}$ of pepsin $(5 \mathrm{mg} / \mathrm{ml}$ in $0.1 \mathrm{~N} \mathrm{HCl} / 0.9 \% \mathrm{NaCl}$; Sigma, St Louis, MO, USA) at $37^{\circ} \mathrm{C}$ for $40 \mathrm{~min}$. The slides were rinsed with distilled water for $3 \mathrm{~min}$, washed with $2 \times$ standard saline citrate for $5 \mathrm{~min}$ and air dried. Probes for enumeration of CEP 7, CEP 17, and CEP Y were from Vysis (Abbott, Downers Grove, IL, USA).

The alterations in chromosomes 7 and 17 were assessed using a probe cocktail containing probe CEP7 (green) and CEP17 (orange). The CEP7/CEP17 probe set and CEP Y probe were diluted with tDenHyb2 (Insitus, Albuquerque, NM, USA) in ratios of 1:50 and 1:100, respectively. The method of analysis has been partially described previously. ${ }^{13-18}$ In brief, for each slide, 100-150 nuclei from tumor tissue were scored for probe signals under the fluorescence microscope with $\times 1000$ magnification. Definitions of chromosomal trisomy for chromosomes 7,17 , and $\mathrm{Y}$ deletion were based on the Gaussian model and were related to the non-neoplastic renal cortex control cell signals. The cutoff values were set for each probe at the mean +3 s.d. of the control values. This research was approved by the Institutional Review Board.

\section{Results}

We analyzed 37 tumors originally diagnosed as metanephric adenomas (2 of which we reclassified as papillary renal cell carcinomas). Among those 35 metanephric adenomas, there were 23 women and 12 men (F:M, 1.9:1) who ranged in age from 7 to 84 years (mean, 51 years). Tumors ranged in size from 1.1 to $7.0 \mathrm{~cm}$. Histologically, all neoplasms showed a fairly well-circumscribed mass composed of small, uniform, epithelial cells that formed small acini separated by hypocellular stroma (Figures 1a-d). The frequently overlapping tumor cells had scant pale-staining cytoplasm, dark nuclei, and inconspicuous nucleoli. Mitotic figures were not seen. Patient and tumor characteristics are summarized in Table 2.

Most of the tumors originally diagnosed as metanephric adenomas (31/37) showed the expected staining pattern: CK7-, AMACR-, WT1+, and CD57+ (Figures 1e-h; Tables 2 and 3). Six tumors $(16 \%)$ exhibited a discordant immunophenotype. Of tumors that deviated from this staining pattern, one yielded the opposite results (CK7+, AMACR+, WT1-, CD57-; Figure 2) and another was positive for CK7, AMACR, and focally CD57 positive but 
negative for WT1 (Figure 3). A third tumor was positive for WT1, AMACR, and CD57, and negative for CK7. Three tumors showed membranous rather than cytoplasmic positivity for CD57 (Figure 4).
Immunoreactivity for AMACR and CK7 was present in $<1 \%$ of cells in three and four tumors, respectively, which we interpreted as negative. Immunohistochemical results are summarized in Tables 2 and 3.
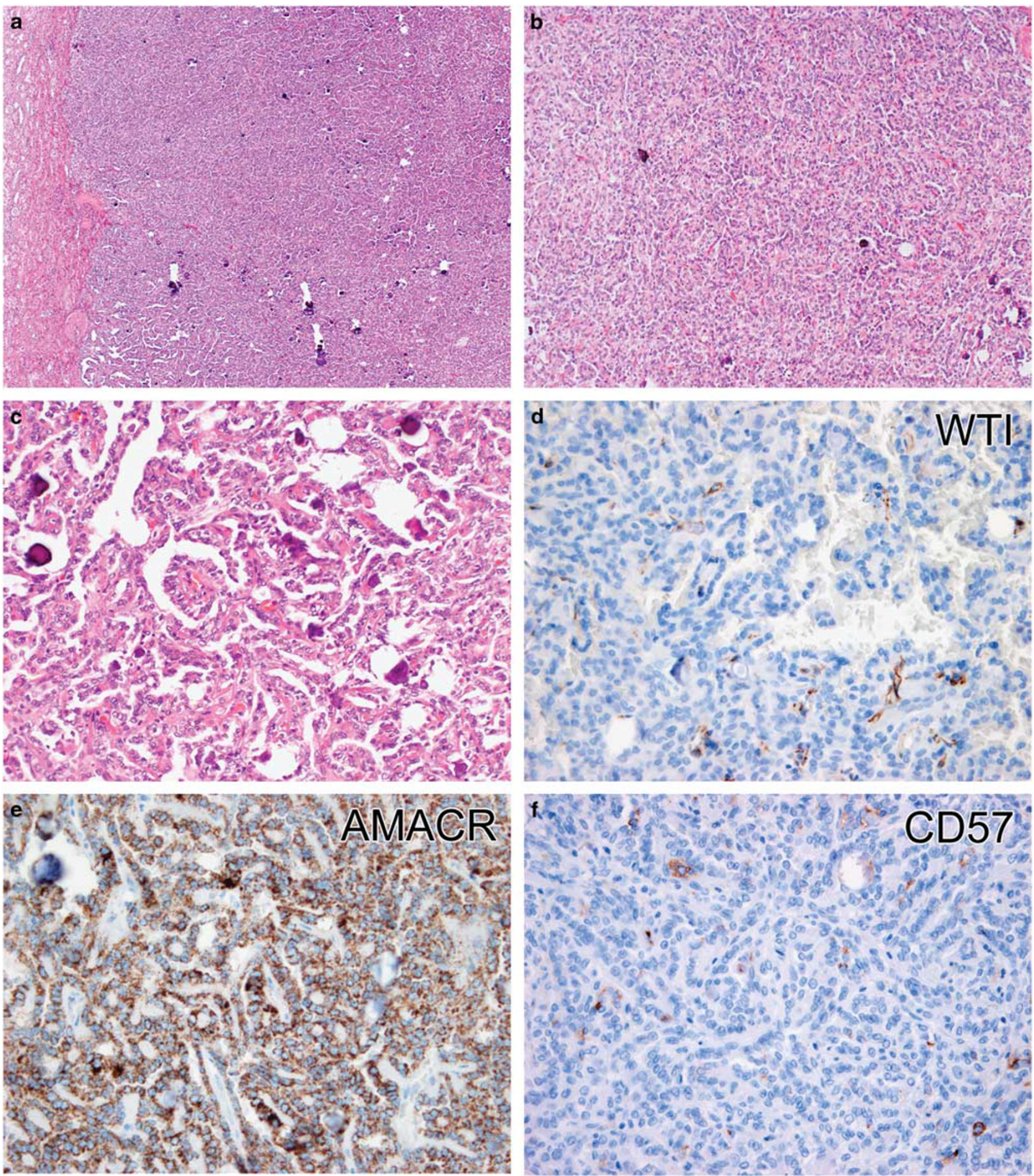

Figure 2 Histology and immunohistochemistry of Case A (\#36) reclassified as papillary renal cell carcinoma. (a) Distinct interface between tumor and uninvolved renal parenchyma. (b) More solid area of ill-defined tubules and dystrophic calcification. (c) Glomeruloid bodies and dystrophic calcification. (d) WT1 is negative. (e) AMACR shows cytoplasmic positivity. (f) CD57 is negative. (g) CK7 shows cytoplasmic positivity. (h) Fluorescence in situ hybridization with centromeric probe for chromosome Y (green signal) showed that green signals only exist in two stromal cells demonstrating deletion of Y chromosome in tumor cells. 

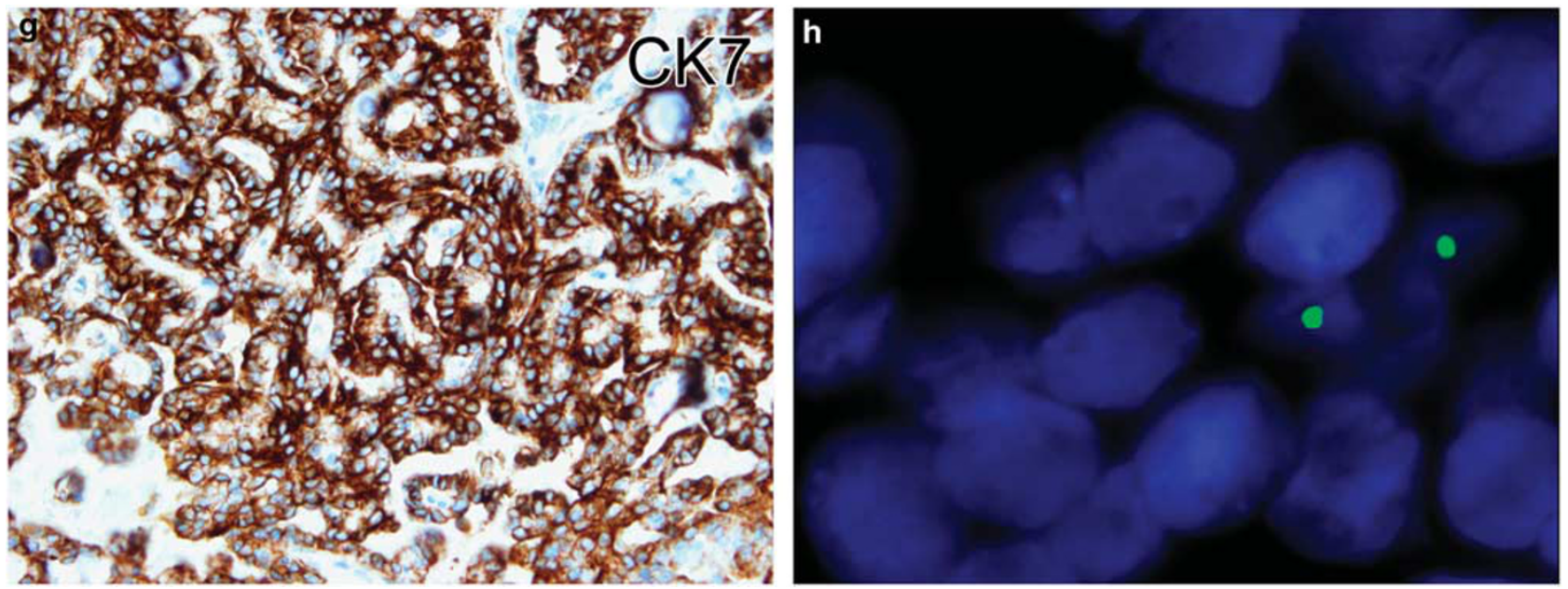

Figure 2 Continued.

Of the six tumors with discordant immunohistochemical staining patterns, the first (CK7+, AMACR+, WT1-, CD57-) exhibited trisomy of chromosomes 7 and 17 and loss of $\mathrm{Y}$ by FISH. The second (CK7+, AMACR+, CD57+, WT1-) demonstrated loss of Y only. Based on these immunohistochemical and molecular profiles, we reclassified these two tumors as papillary renal cell carcinomas. The remaining tumors with discordant immunohistochemical findings demonstrated disomy of chromosomes 7,17 , and no deletion of $\mathrm{Y}$, supporting the original classification as metanephric adenoma. FISH results are summarized in Table 2.

We analyzed 13 solid variant papillary renal cell carcinomas (plus the 2 reclassified tumors). All but two tumors were positive for AMACR and CK7 and negative for WT1 and CD57 (Table 3; Figure 5). One tumor was focally positive for CD57 and one tumor was negative for CK7. FISH results revealed five tumors with deletion of $\mathrm{Y}$, three with trisomy of chromosomes 7 and 17, four with trisomy of 7,17 , and deletion of $\mathrm{Y}$, and one with no chromosomal aberrations.

We also analyzed 20 epithelial-predominant nephroblastomas. All were positive for WT1 and negative for AMACR (Table 3; Figure 6). One tumor was positive for CK7. Sixteen tumors were positive for CD57. FISH results revealed all tumors had disomy of chromosomes 7 and 17 and no deletion of $\mathrm{Y}$ in male cases. When comparing metanephric adenoma with the solid variant of papillary renal cell carcinoma, the sensitivities of WT1, CK7, and CD57 were $100 \%$ and of AMACR was $97 \%$ (Tables 3 and 4). Specificities of WT1 and AMACR were 100\% and of CK7 and CD57 were 93\%. When comparing metanephric adenoma with epithelial-predominant nephroblastoma, WT1 and AMACR were $0 \%$, CK7 $5 \%$, and CD57 were $20 \%$ specific (Tables 3 and 4$)$.

\section{Discussion}

Metanephric adenoma is a benign renal neoplasm with a differential diagnosis that can essentially be categorized based on age. In children (12 years and younger), metanephric adenoma overlaps in morphology with epithelial-predominant nephroblastoma. In adults ( $\geq 30$ years), metanephric adenoma can have morphology similar to papillary renal cell carcinoma. In our study, we report only one case of a pediatric metanephric adenoma, and therefore, our study only addresses metanephric adenomas in the adult population. Previous studies have helped distinguish between these diagnoses with immunohistochemistry; however, there are still cases that deviate from the expected immunohistochemical patterns. To help establish a definitive diagnosis, we investigated the utility of combined immunohistochemical and molecular analyses of 37 tumors originally diagnosed as metanephric adenomas (2 tumors we reclassified as papillary renal cell carcinomas), 13 solid variant papillary renal cell carcinomas (plus the 2 reclassified tumors), and 20 epithelial-predominant nephroblastomas; the first study, to our knowledge, to use a combined approach for all three tumor types. Of the total 37 tumors originally diagnosed as metanephric adenomas, we identified six tumors with discordant immunohistochemical staining patterns and two of those tumors we reclassified as papillary renal cell carcinomas based on FISH results of trisomy of chromosomes 7 and 17 and loss of Y. Our data suggest the combination approach of immunohistochemical analysis and FISH studies in handling certain difficult cases.

Most renal tumors can be diagnosed based on morphology in routine sections; however, there are certain diagnoses with overlapping features. Metanephric adenoma, an uncommon benign tumor, can show morphological similarities to some papillary renal cell carcinomas and epithelial-predominant 


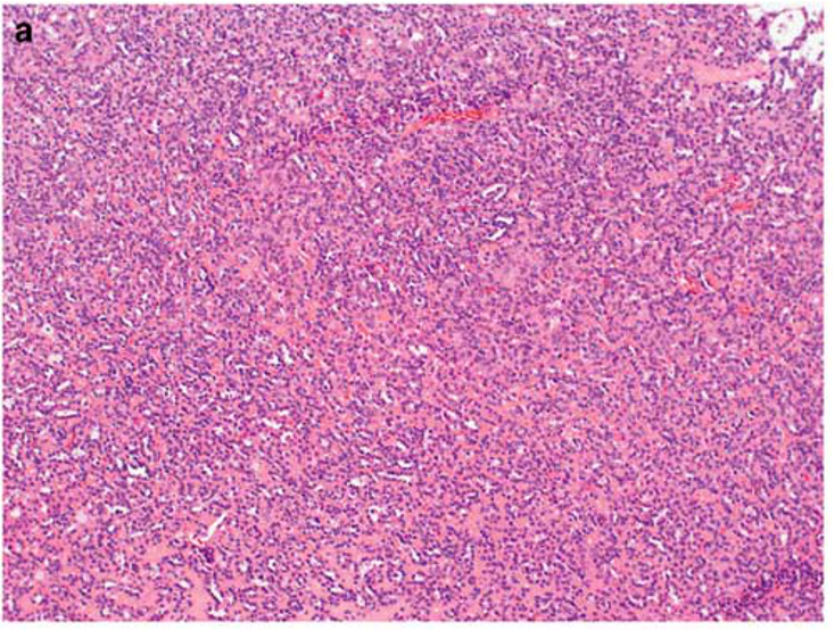

cop ${ }^{2}$.

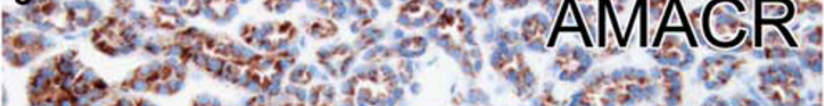

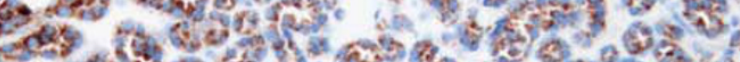

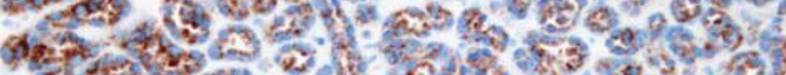

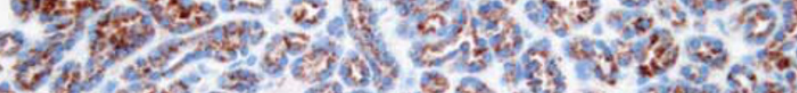

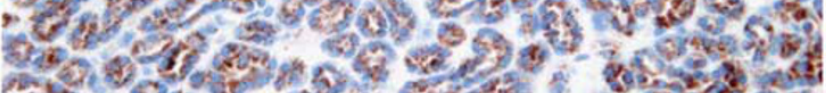

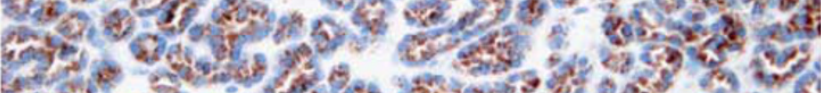
c4.

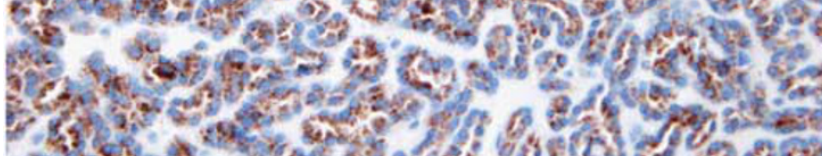

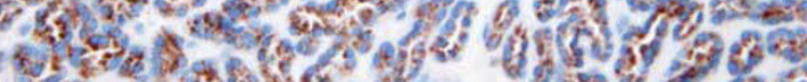

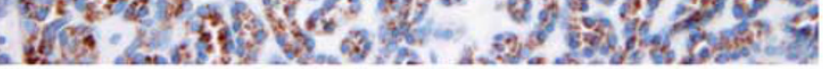

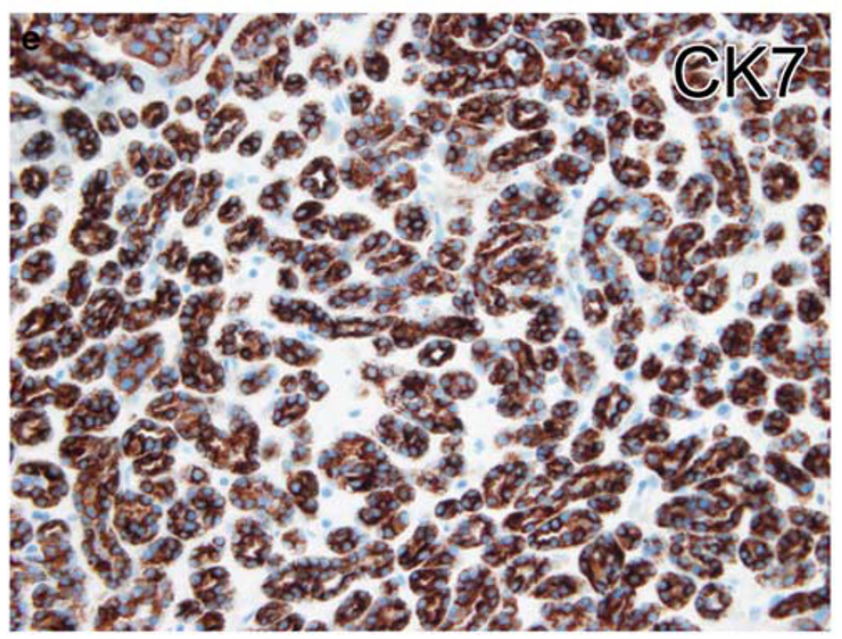

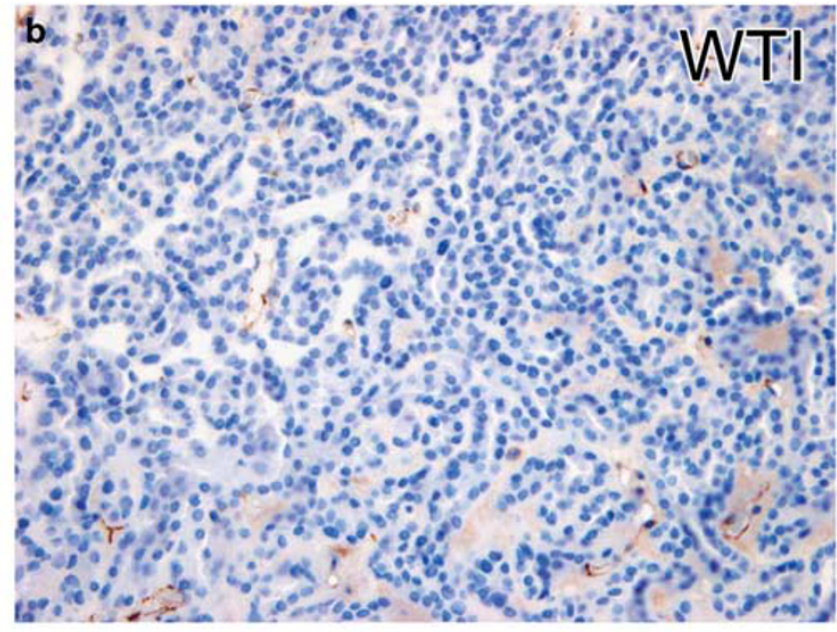
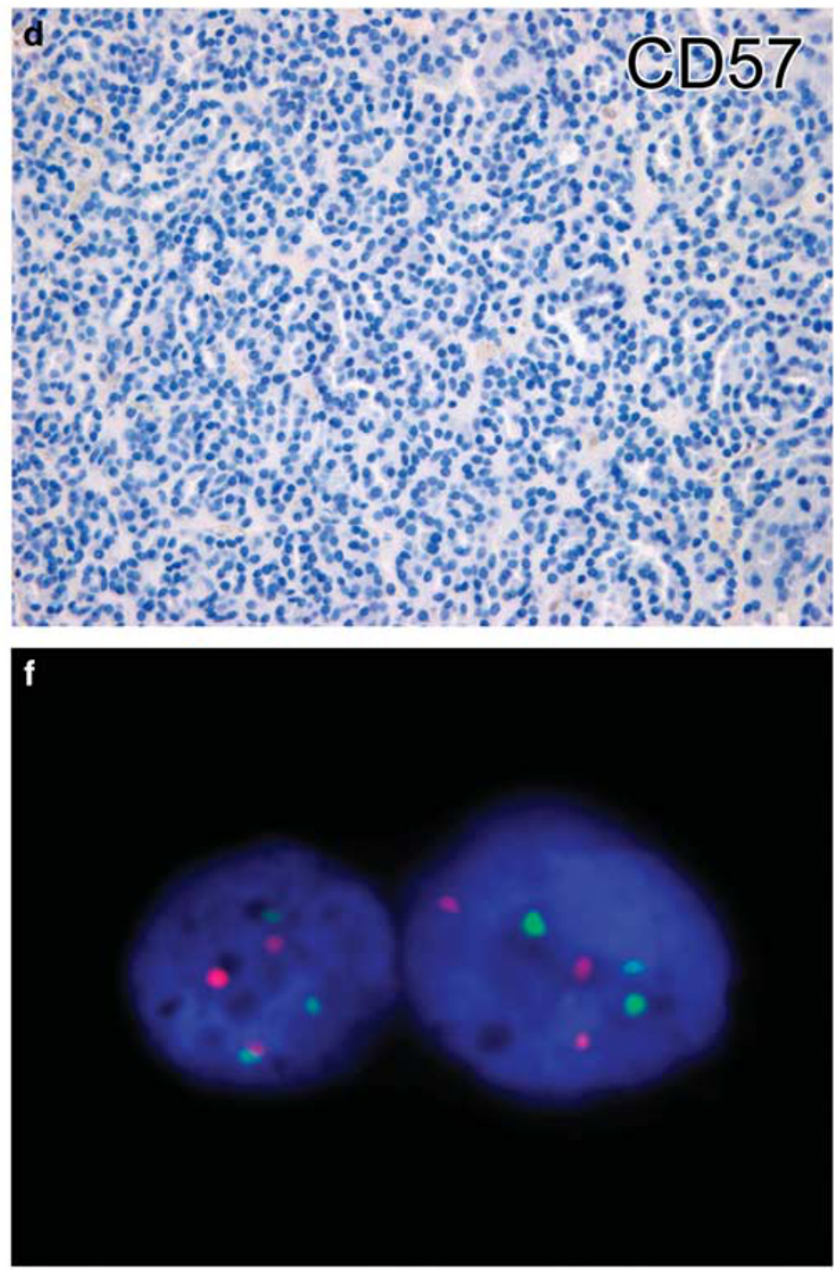

Figure 3 Histology and immunohistochemistry of Case B (\#37) reclassified as papillary renal cell carcinoma. (a) Acini with intervening edematous stroma. (b) WT1 is negative. (c) AMACR shows cytoplasmic positivity. (d) CD57 is negative. (e) CK7 shows cytoplasmic positivity. (f) Fluorescence in situ hybridization with centromeric probes 7 (red) and 17 (green) showed tumor nuclei containing three red and three green signals demonstrating trisomy of chromosomes 7 and 17.

nephroblastoma, both malignant neoplasms. Morphologically, papillary renal cell carcinomas are composed predominantly of papillary or tubulopapillary structures lined by either small or larger cells; however, compression of the papillary structures can result in more solid architectural patterns. ${ }^{19}$ It is in these instances that the differential diagnosis includes metanephric adenoma. 
Microscopically, most nephroblastomas are triphasic, containing blastemal cells and epithelial and stromal elements. ${ }^{20}$ Epithelial components can include primitive rosette-like structures and tubular or papillary elements and consequently can be difficult to differentiate morphologically from metanephric adenomas and papillary renal cell carcinomas. ${ }^{6}$ To distinguish between these entities is important for treatment and follow-up of patients.

Similar to previous studies, excluding the two cases reclassified as papillary renal cell carcinoma, all of our 35 metanephric adenomas were positive for WT1 and negative for CK7. In comparison to other cases, Muir et $a l^{5}$ showed that all 6 of their cases of metanephric adenoma were strongly and diffusely positive for WT1 and some focally positive with antibodies to CK7, defined as staining of $<5 \%$ of the tumor cells. Olgac et $a l^{6}$ showed that 7 out of 10 metanephric adenomas were positive for WT1 and all were negative for CK7. These results slightly differ from ours and could be due to the use of different antibodies. Olgac et $a l^{6}$ used WT1 from Santa Cruz, CA, USA at a 1:4000 dilution. Also, their cutoff for positivity was $5 \%$ versus $1 \%$ in ours. In addition, $100 \%$ (35/35) of our metanephric adenomas, all 21 cases from Mantoan Padilha et al, ${ }^{21}$ all 6 cases from Muir et al, ${ }^{5}$ and 6 out of 10 cases from

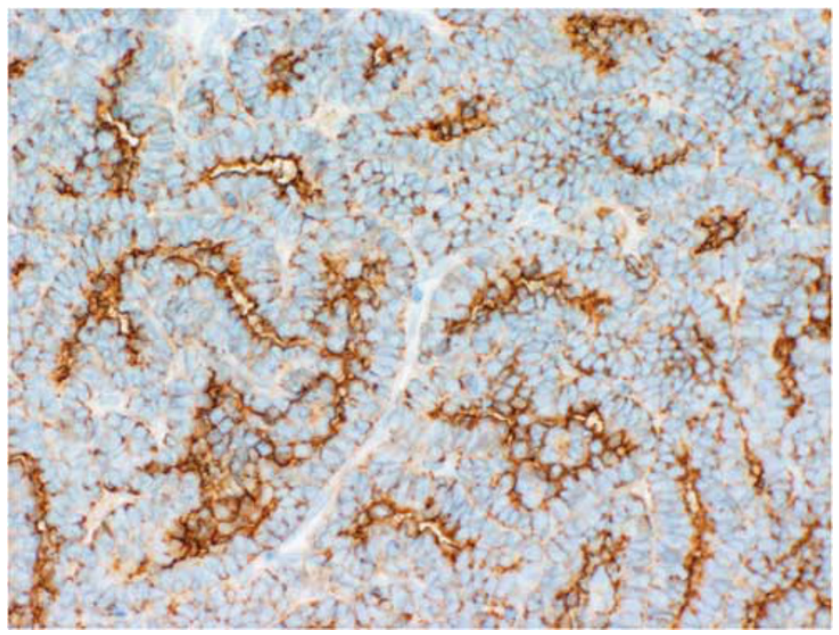

Figure 4 Membranous staining of CD57 in metanephric adenoma.
Olgac et $a l^{6}$ stained positive for CD57 (including membranous staining).

In keeping with Olgac et $a l^{6}$ who showed that AMACR was positive in one of 10 metanephric adenomas, 24 of the 25 papillary renal cell carcinomas, and negative in all 8 nephroblastomas, we also found AMACR positive in 1 metanephric adenoma, positive in both cases reclassified as papillary renal cell carcinoma, positive in all the other 13 papillary renal cell carcinomas, and negative in all 20 epithelial-predominant nephroblastomas. Mantoan Padilha et $a l^{21}$ also demonstrated $5 \%$ of 21 metanephric adenomas and $100 \%$ of 23 papillary renal cell carcinomas positive for AMACR.

Of note, Azabdaftari et $a^{22}$ demonstrated strong expression of $\mathrm{S} 100$ protein in 15 metanephric adenomas and no expression in 13 papillary renal cell carcinomas. In contrast, Mantoan Padilha et al ${ }^{21}$ detected S100 staining in all 21 metanephric adenomas and $22 \%$ of papillary renal cell carcinomas. Other studies have described negative staining for S100 in metanephric adenomas. ${ }^{3}$ Because of this discrepancy, we elected not to include S100 in our immunohistochemical panel.

There are still cases of metanephric adenomas, as demonstrated in both studies by Muir et $a l^{5}$ and Olgac et $a l^{6}$ and six in our study, which deviate from the expected immunohistochemical staining pattern, including membranous CD57 staining and positive AMACR staining. In these instances, we investigated the utility of FISH analysis in establishing the diagnosis. In the current study, two of the six cases that were analyzed with FISH showed gains of chromosomes 7 and 17 and/or the loss of chromosome Y. Thus these cases were reclassified as papillary renal cell carcinomas. Brunelli et $a l^{9}$ previously demonstrated in a study of seven metanephric adenomas that these tumors are cytogenetically similar to normal kidney and lack the gains of chromosomes 7 and 17 and loss of $Y$ that are typical of papillary renal cell neoplasms.

In summary, to help establish a definitive diagnosis of metanephric adenoma, a benign tumor, from papillary renal cell carcinoma and epithelialpredominant nephroblastoma, we investigated the utility of combined immunohistochemical and molecular analyses of 37 tumors originally diagnosed as

Table 4 Typical immunohistochemical and cytogenetic profiles of metaphoric adenoma, epithelial-predominant nephroblastoma, and solid variant papillary renal cell carcinoma

\begin{tabular}{llll}
\hline Markers & Metanephric adenoma & Epithelial-predominant nephroblastoma & Solid variant papillary renal cell carcinoma \\
\hline AMACR & Negative & Negative & Positive \\
WT1 & Positive & Positive & Negative \\
CK7 & Negative & Negative & Positive \\
CD57 & Positive & Negative or focal & Negative \\
Loss of Y chromosome & Negative & Negative & Positive \\
Trisomy $7 / 17$ & Negative & Negative & Positive \\
\hline
\end{tabular}



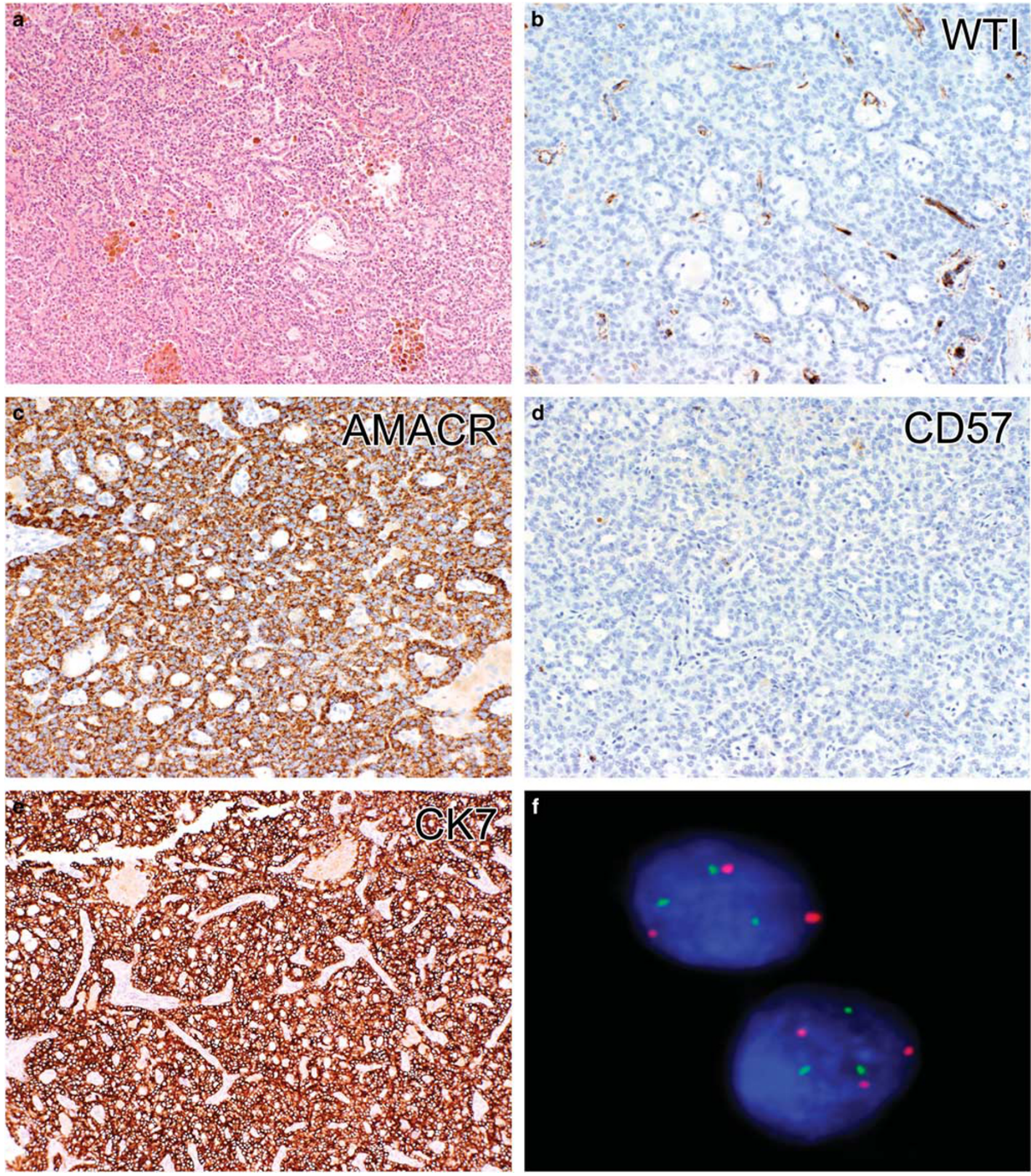

Figure 5 Histology, immunohistochemistry, and fluorescence in situ hybridization (FISH) of solid variant papillary renal cell carcinoma. (a) Papillary structures and tightly packed acini. (b) WT1 is negative. (c) AMACR shows cytoplasmic positivity. (d) CD57 is negative. (e) CK7 shows cytoplasmic positivity. (f) FISH with centromeric probes 7 (red) and 17 (green) showed tumor nuclei containing three red and three green signals demonstrating trisomy of chromosomes 7 and 17.

metanephric adenoma (2 tumors we reclassified as papillary renal cell carcinomas), 13 solid variant papillary renal cell carcinomas (plus the 2 reclassified tumors), and 20 epithelial-predominant nephro- blastomas. Six of these tumors (16\%) deviated from the previously reported and expected immunohistochemical staining profile for metanephric adenoma (CK7-, AMACR-, WT1+, and CD57+). Two of those 

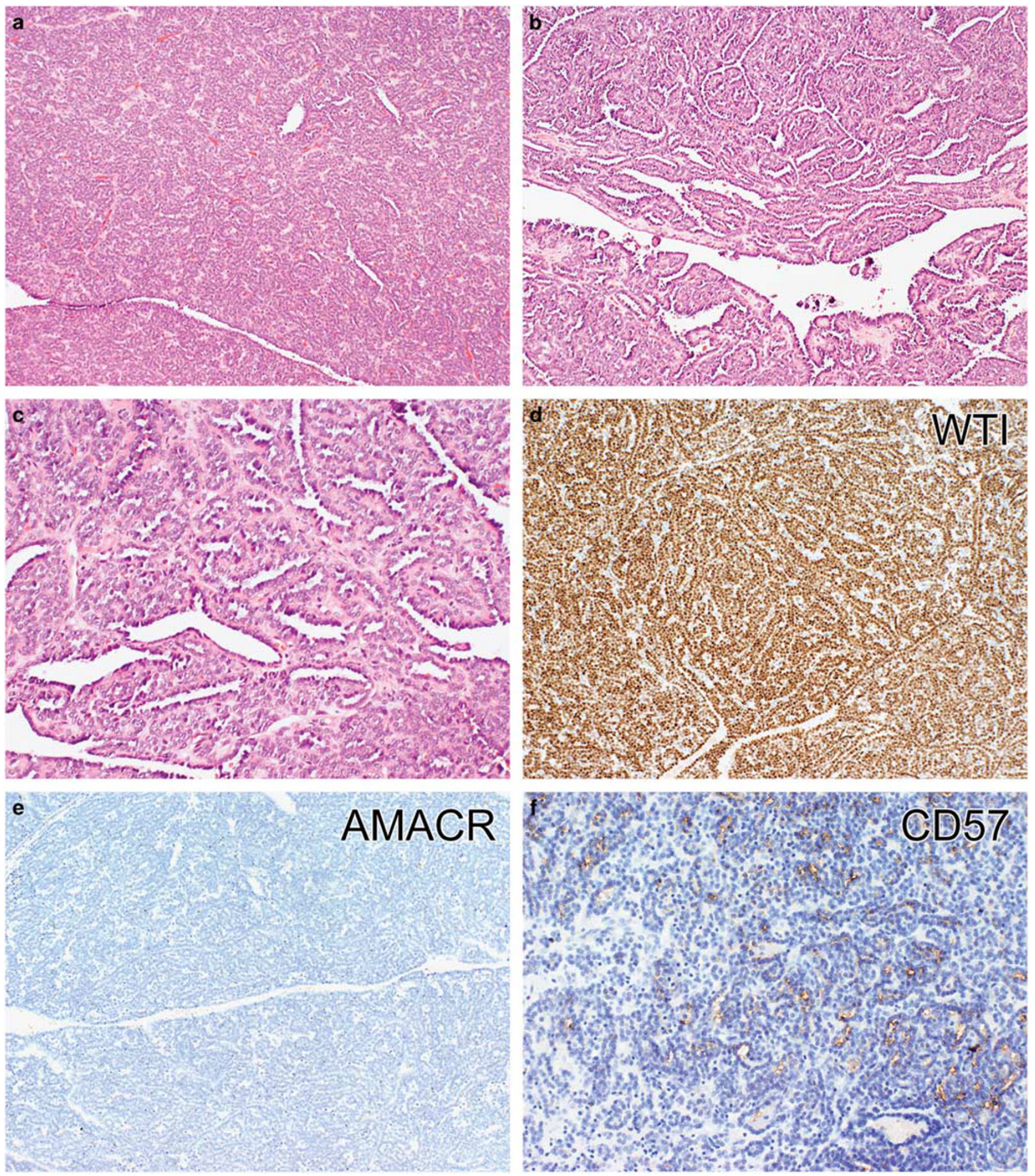

Figure 6 Histology, immunohistochemistry, and fluorescence in situ hybridization (FISH) of epithelial-predominant nephroblastoma. (a) Tightly packed ill-defined tubules. (b) Papillary structures. (c) Ill-defined tubules with slightly edematous stroma. (d) WT1 shows nuclear positivity. (e) AMACR is negative. (f) CD57 is focally positive. (g) CK7 is negative. (h) FISH with centromeric probes 7 (red) and 17 (green) showed tumor nuclei containing two red and two green signals demonstrating normal disomy of chromosomes 7 and 17 .

tumors we reclassified as papillary renal cell carcinomas based on FISH results characteristic of papillary renal cell carcinomas (trisomy of chromosomes 7 and 17 and loss of Y). FISH analysis suggests that immunohistochemistry appears to be sufficient in establishing the diagnosis of metanephric adenoma in the majority of cases. It is recommended that all tumors in adults histologically resembling 

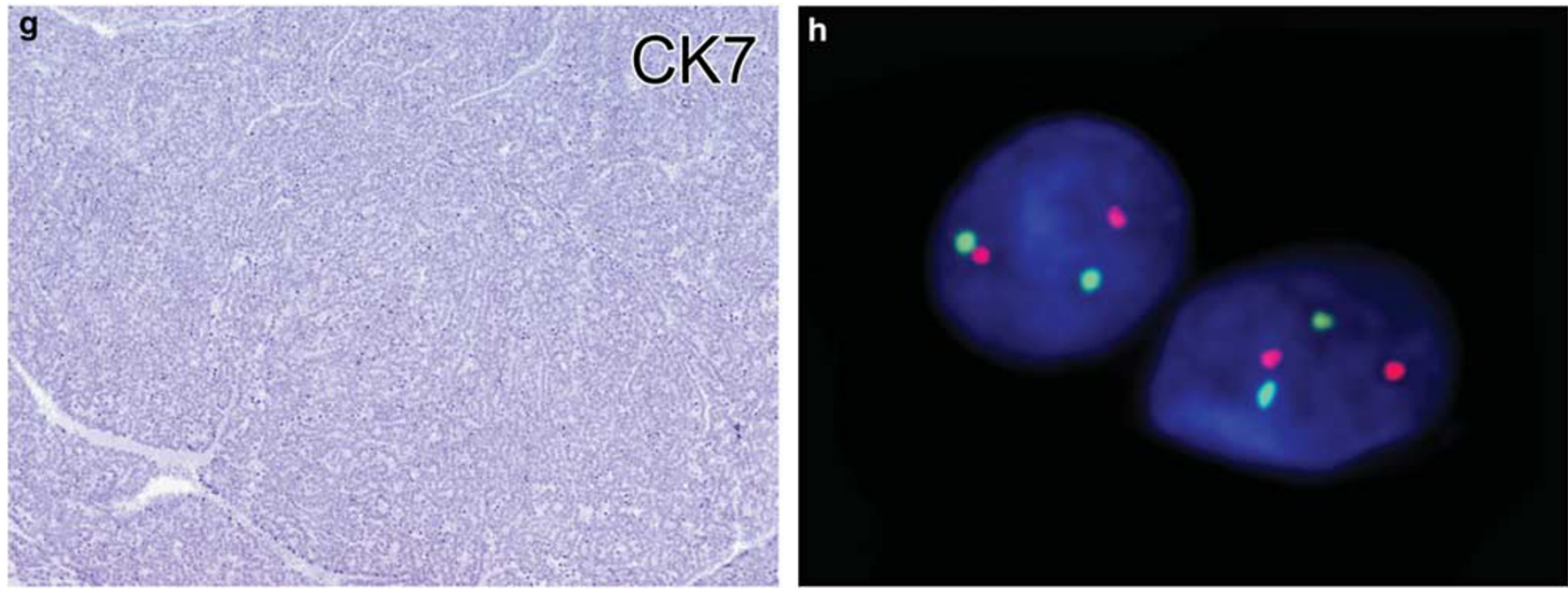

Figure 6 Continued.

metanephric adenoma have WT1, CD57, CK7, and AMACR immunohistochemical staining performed. If the staining pattern is characteristic for metanephric adenoma (CK7-, AMACR-, WT1+, and CD57+, including membranous CD57 staining), then no other diagnostic tests are indicated. However, if there is a different immunostaining pattern, then we recommend FISH analysis in further diagnostic workup.

\section{Disclosure/conflict of interest}

The authors declare no conflict of interest.

\section{References}

1 Davis CJ Jr, Barton JH, Sesterhenn IA et al. Metanephric adenoma. Clinicopathological study of fifty patients. Am J Surg Pathol 1995;19:1101-1114.

2 Gatalica Z, Grujic S, Kovatich A et al. Metanephric adenoma: histology, immunophenotype, cytogenetics, ultrastructure. Mod Pathol 1996;9:329-333.

3 Jones EC, Pins M, Dickersin GR et al. Metanephric adenoma of the kidney. A clinicopathological, immunohistochemical, flow cytometric, cytogenetic, and electron microscopic study of seven cases. Am J Surg Pathol 1995;19:615-626.

4 Bruder E, Passera O, Harms D et al. Morphologic and molecular characterization of renal cell carcinoma in children and young adults. Am J Surg Pathol 2004;28: 1117-1132.

5 Muir TE, Cheville JC, Lager DJ. Metanephric adenoma, nephrogenic rests, and Wilms' tumor: a histologic and immunophenotypic comparison. Am J Surg Pathol 2001;25:1290-1296.

6 Olgac S, Hutchinson B, Tickoo SK et al. Alphamethylacyl-CoA racemase as a marker in the differential diagnosis of metanephric adenoma. Mod Pathol 2006;19:218-224.

7 Charles AK, Mall S, Watson J et al. Expression of the Wilms' tumour gene WT1 in the developing human and in paediatric renal tumours: an immunohistochemical study. Mol Pathol 1997;50:138-144.

8 Ramani P, Cowell JK. The expression pattern of Wilms' tumour gene (WT1) product in normal tissues and paediatric renal tumours. J Pathol 1996;179: 162-168.

9 Brunelli M, Eble JN, Zhang S et al. Metanephric adenoma lacks the gains of chromosomes 7 and 17 and loss of $\mathrm{Y}$ that are typical of papillary renal cell carcinoma and papillary adenoma. Mod Pathol 2003;16:1060-1063.

10 Corless CL, Aburatani H, Fletcher JA et al. Papillary renal cell carcinoma: quantitation of chromosomes 7 and 17 by FISH, analysis of chromosome $3 p$ for LOH, and DNA ploidy. Diagn Mol Pathol 1996;5:53-64.

11 Kattar MM, Grignon DJ, Wallis T et al. Clinicopathologic and interphase cytogenetic analysis of papillary (chromophilic) renal cell carcinoma. Mod Pathol 1997;10:1143-1150.

12 Kovacs G, Fuzesi L, Emanual A et al. Cytogenetics of papillary renal cell tumors. Genes Chromosomes Cancer 1991;3:249-255.

13 Cheng L, MacLennan GT, Zhang S et al. Evidence for polyclonal origin of multifocal clear cell renal cell carcinoma. Clin Cancer Res 2008;14:8087-8093.

14 Cheng L, Zhang S, MacLennan GT et al. Molecular and cytogenetic insights into the pathogenesis, classification, differential diagnosis, and prognosis of renal epithelial neoplasms. Hum Pathol 2009;40:10-29.

15 Gobbo S, Eble JN, MacLennan GT et al. Renal cell carcinomas with papillary architecture and clear cell components: the utility of immunohistochemical and cytogenetical analyses in differential diagnosis. Am J Surg Pathol 2008;32:1780-1786.

16 Jones TD, Eble JN, Wang $\mathrm{M}$ et al. Molecular genetic evidence for the independent origin of multifocal papillary tumors in patients with papillary renal cell carcinomas. Clin Cancer Res 2005;11:7226-7233.

17 Williamson SR, Zhang S, Eble JN et al. Clear cell papillary renal cell carcinoma-like tumors in patients with von Hippel-Lindau disease are unrelated to sproadic clear cell papillary renal cell carcinoma. Am J Surg Path 2013;37:1131-1139.

18 Halat S, Eble JN, Grignon DJ et al. Multilocular cystic renal cell carcinoma is a subtype of clear 
cell renal cell carcinoma. Mod Pathol 2010;23: 931-936.

19 Lager DJ, Huston BJ, Timmerman TG et al. Papillary renal tumors. Morphologic, cytochemical, and genotypic features. Cancer 1995;76:669-673.

20 Sebire NJ, Vujanic GM. Paediatric renal tumours: recent developments, new entities and pathological features. Histopathology 2009;54:516-528.
21 Mantoan Padilha M, Billis A, Allende D et al. Metanephric adenoma and solid variant of papillary renal cell carcinoma: common and distinctive features. Histopathology 2013;62:941-953.

22 Azabdaftari G, Alroy J, Banner BF et al. S100 protein expression distinguishes metanephric adenomas from other renal neoplasms. Pathol Res Pract 2008;204: 719-723. 